\title{
Separating the Vibrationally Resolved Auger Decay Channels for a CO Core Hole State
}

\author{
V. Ulrich, S. Barth, S. Joshi, T. Lischke, A. M. Bradshaw, and U. Hergenhahn* \\ Max-Planck-Institut für Plasmaphysik, EURATOM Association, Boltzmannstr. 2, 85748 Garching, Germany
} (Received 14 May 2007; published 10 April 2008)

\begin{abstract}
The $K-V V$ Auger spectrum of carbon monoxide (CO) excited by $\mathrm{C} 1 s$ photoionization has been investigated with a novel electron-electron coincidence setup. The energy resolution is sufficiently high to resolve the vibrational energy levels of the core-ionized intermediate state and of most dicationic final states in the two-dimensional electron energy map. We demonstrate how the influence of vibrational states on a molecular Auger spectrum can be accessed experimentally without the constraint of averaging over all intermediate state energies.
\end{abstract}

DOI: 10.1103/PhysRevLett.100.143003

PACS numbers: 33.60.+q, 07.81.+a, 33.70.Ca, 33.80.Eh

Although the quantitative description of molecular orbitals and nuclear configuration began 70 years ago, it still poses a great challenge to theorists. Diatomic molecules are the simplest systems and many sophisticated calculations have been performed for neutral and ionized species. Experimental data on molecular ions provide a good test for the theory of molecular states, since predicting the properties of these systems is much more challenging than that for the neutral ground state. While photoelectron spectroscopy provides detailed information about the electronic [1] and vibrational [2] structure of singly ionized molecules, Auger electron spectroscopy has so far only been able to provide this information for doubly ionized (dicationic) molecules in a limited way. This is because the Auger lines are usually numerous and overlapping and thus cannot be assigned unambiguously. Moreover, the combined influence of the vibrational structure in the intermediate and final states is typically subsumed in current Auger theory by a shift and a Gaussian broadening of the electronic transitions [3]. Only in exceptional cases has it been disentangled by a numerical algorithm that simulates the joint photoionization + Auger decay transition amplitude based on parametrized potential curves of the participating states [4,5]. In the second of these two papers the authors have presented a simulated photoelectron-Auger electron coincidence spectrum. Our experiment uses Auger electron-photoelectron coincidence spectroscopy to assign experimentally the Auger electrons to their associated photoelectrons and is thus the first direct experimental test of the model calculations of Püttner et al. [5].

In this Letter we report photoelectron-Auger electron coincidence measurements on carbon monoxide (CO) after $\mathrm{C} 1 s$ ionization by synchrotron radiation. Our energy resolution allows the vibrational structure of the intermediate $\mathrm{C}$ $1 s^{-1}$ state and of most of the final $\mathrm{CO}^{2+}$ states to be resolved. We can thus identify the channels for the population of the dicationic final states in their respective vibrational substates starting from the core-ionized intermediate state, and thus explain the structure of the Auger spectrum. These studies have become possible because of a novel coincidence detection setup, in which a high resolution electrostatic analyzer is coupled to a number of time-offlight spectrometers. As a result, we have been able to detect both electrons simultaneously within significant kinetic energy intervals and with good energy resolution.

The inner shell photoionization [6] and the subsequent Auger decay $[7,8]$ of $\mathrm{CO}$ has been intensively studied, i.e., by high resolution electron spectroscopy, $e^{-}$, $e^{-}$-coincidence spectroscopy at lower energy resolution [9] and recently by electron-ion coincidence methods $[10,11]$. The potential curves of such final (dicationic) states have been investigated theoretically and, using photo-double-ionization, experimentally by Eland et al. [12]. All lower singlet states and most lower triplet states have local minima, which means they support metastable states, the lifetimes of which are still the subject of discussion [12,13], but are sufficient to justify the notion of vibrational structure in the final state. The Auger spectrum has been assigned to the different asymptotic $\mathrm{C}^{+}+\mathrm{O}^{+}$ channels in electron-ion coincidence experiments [11]. Questions remained, however, as to the assignment of the spectrum to the different dicationic states [8]. In contrast to the experiments using photo-double-ionization, in the present study the same final states can be populated from different vibrational $\mathrm{CO}^{+}$intermediate states. Thus, our experiment is sensitive not only to the spectroscopic parameters of the final state, but also to the relative position of the intermediate and final state potential curves. Photoelectron-Auger electron coincidence spectroscopy has been pioneered by Haak et al. [14], and first results for free molecules were obtained by Neeb et al. [15] for $\mathrm{N}_{2}$, but without vibrational resolution.

The electronic configuration in the ground state of $\mathrm{CO}$ is $1 s_{O}^{2} 1 s_{C}^{2} 3 \sigma^{2} 4 \sigma^{2} 1 \pi^{4} 5 \sigma^{2}$, with an internuclear distance of $R_{e}=0.1128 \mathrm{~nm}$ [16]. By C $1 s$ photoionization the molecule transits to a highly excited state of $\mathrm{CO}^{+*}$, the vibrational structure of which is imprinted on the photoelectrons ([2], see left-hand side histogram in Fig. 1). The ionization leads to a bond shortening of $4.9 \mathrm{pm}$, and the vibrational energy in the $\mathrm{C} 1 s^{-1}$ state is $304 \mathrm{meV}[2,17]$. Subsequent 


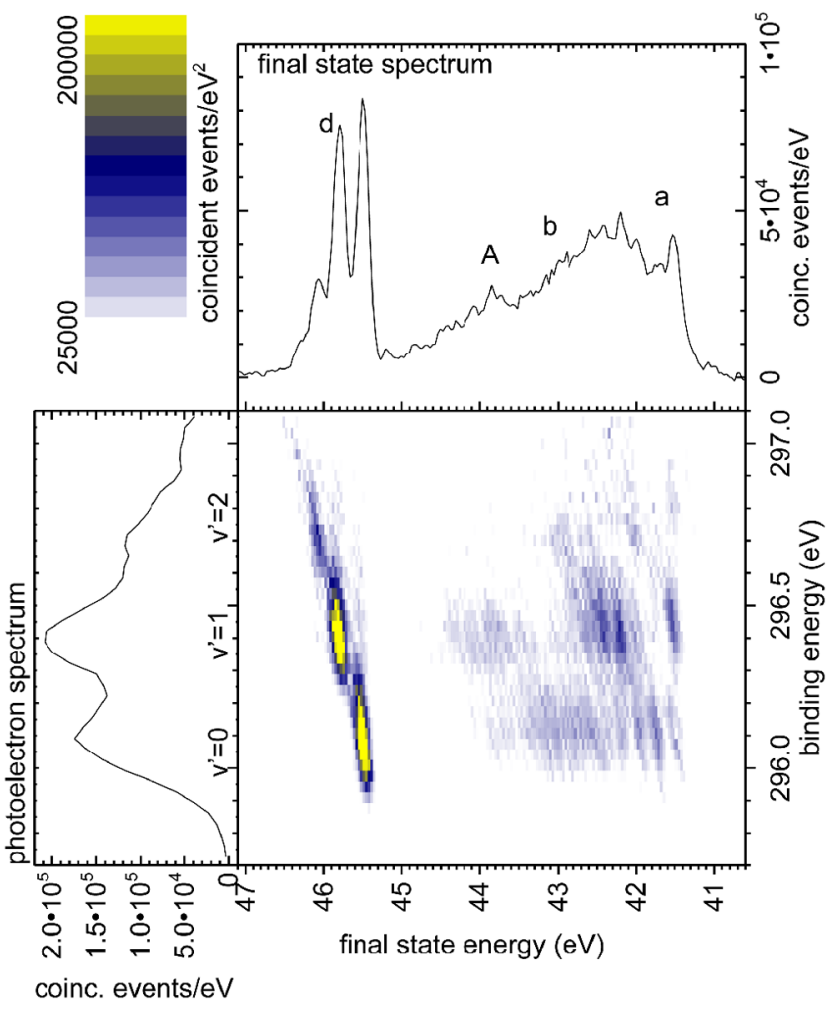

FIG. 1 (color online). Photoelectron-Auger electron coincidence spectrum of $\mathrm{CO}$ after $\mathrm{C} 1 s$ ionization at $304 \mathrm{eV}$ photon energy. For each combination of $\mathrm{CO}^{+}$intermediate and $\mathrm{CO}^{2+}$ final states the number of electron pairs recorded is plotted on a color-coded map. The contribution of random coincidences has been subtracted [21]. Intensities are given as events per unit energy and events per unit energy ${ }^{2}$, respectively.

Auger decay populates several dissociative and metastable final states with different internuclear distances. The energy $E_{\mathrm{fi}}$ of a final state can be calculated from the kinetic energy of its Auger electron $E_{\mathrm{au}}$, the photoelectron energy $E_{\mathrm{ph}}$, and the x-ray photon energy by $E_{\mathrm{fi}}=h \nu-E_{\mathrm{au}}-$ $E_{\mathrm{ph}}$. The population of the final states depends essentially on the shape and the relative position of the potential curves involved, expressed in the Franck-Condon principle. By means of our coincidence setup the Auger electron, photoelectron pairs are plotted against their binding energies and in many cases the corresponding final state can be identified. Only regions, in which the vibronic excitations of different final states have similar energies, remain problematic.

The experiment was carried out at beam line UE52SGM [18] of the synchrotron radiation source BESSY (Berlin, Germany) during multibunch operational mode. The beam line resolution was set to $90 \mathrm{meV}$. An effusive beam of $\mathrm{CO}$ was used, with the chamber pressure at about $3 \times 10^{-6}$ mbar. Photoelectrons were recorded by five identical time-of-flight spectrometers (TOF) mounted in the plane spanned by the light propagation axis and the (vertical) electric field vector of the synchrotron radiation.
Each TOF consists of a grounded $4 \mathrm{~mm}$ aperture, a first retarding tube of conical shape, a cylindrical second retarding tube, and a stack of three microchannel plates. Setting suitable retarding voltages we have obtained a total resolution of $200 \mathrm{meV}$ and a transmission variation of less than $10 \%$ for photoelectrons of the chosen energy. Times of flight in the TOF spectrometers were converted to energies using reference measurements. Auger electrons were measured with a hemispherical detector (Scienta ES 200 ), mounted in the dipole plane under an angle of $35.3^{\circ}$ with respect to the polarization vector, at a pass energy of $75 \mathrm{eV}$. The energy resolution amounted to approx. $200 \mathrm{meV}$. The Scienta analyzer was equipped with a delay line anode (Roentdek) to allow the separation of coincident events [19]. Electron energies were calibrated to an adiabatic $\mathrm{C} 1 s$ ionization potential of $296.07 \mathrm{eV}$ [20] and a final state energy of $45.461 \mathrm{eV}$ for the $d\left(2^{1} \Sigma^{+}, \nu^{\prime \prime}=0\right)$ level [12]. Further details of the data acquisition will be published separately [21]. About 150000 events, after subtraction of random coincidences, were recorded in $20 \mathrm{~h}$. For this work, signals in all TOF analyzers were summed. As there is no "magic angle" for coincident angular distributions, the results presented inevitably contain a sum of different points in the triple differential cross section, the ratio of which to the total reaction cross section might vary with the reaction channel. In a coordinate system with the polarization vector (oriented vertically) as the polar and the photon momentum as the azimuthal axis, TOF analyzers are at $(\theta, \phi)=\left(0^{\circ}, 0^{\circ}\right),\left(22^{\circ}, 0^{\circ}\right),\left(44^{\circ}, 0^{\circ}\right),\left(22^{\circ}, 180^{\circ}\right)$, $\left(44^{\circ}, 180^{\circ}\right)$ and the Scienta analyzer at $(\theta, \phi)=$ $\left(144.7^{\circ}, 90^{\circ}\right)$.

By virtue of its position-resolving anode, an energy interval of approx. $6.5 \mathrm{eV}$ width can be recorded simultaneously in the electrostatic analyzer at the given settings. In the time-of-flight analyzers, all kinetic energies (larger than $-e$ times the retarding voltage) are detected simultaneously. Resolution and angular acceptance are, however, not homogeneous. Nevertheless, a kinetic energy interval of some $\mathrm{eV}$, as spanned by typical inner shell photoelectron bands [2], can be recorded simultaneously with vibrational resolution. Since the measurements in the TOF analyzers are referred to the arrival times in the hemispherical analyzer, this acquisition scheme is independent of the repetition period of the synchrotron radiation [21]. The time dispersion in the hemispheres makes up the largest contribution to the photoelectron energy uncertainty [19]. The energy resolution achieved nevertheless clearly exceeds that of other schemes for the mapping of two-electron events in energy space [22].

Results of our measurements are shown in Fig. 1. The three most intense dicationic electronic states accessible by Auger decay and observed at the chosen setting of our analyzer are $a\left(1^{1} \Sigma^{+}\right), b\left({ }^{1} \Pi\right)$, and $d\left(2^{1} \Sigma^{+}\right)$. Two structures are immediately apparent in Fig. 1, namely, the "tails" of the $a$ and $d$ states (at the left and right upper 
edge of the map). The long tail of $a$ exhibits the deexcitation from several vibrational intermediate states into the same final state $\nu^{\prime \prime}=0$ with an experimental $E_{\mathrm{fi}}=$ 41.425(10) eV [23] and a calculated equilibrium bond distance of $R_{e}=0.1159 \mathrm{~nm}$ [12], with a change in vibrational quantum number $\Delta \nu$ according to $\Delta \nu=\nu^{\prime}-\nu^{\prime \prime}=$ $0,1,2$ and an intensity maximum for the transition from $\nu^{\prime}=1$. Because the minimum of the potential curve of the $a$ state is shifted from the Franck-Condon (FC) region of $\mathrm{CO}^{+*}$ by $8 \mathrm{pm}$ outwards, not only the ground state $\nu^{\prime \prime}=0$ but also higher vibrational levels $\nu^{\prime \prime}>0$ of $a$ will be populated from $\nu^{\prime}=0$.

This can be seen again in Fig. 2, where the decay intensity of each vibrational intermediate state $\left(\nu^{\prime}=0,1\right.$, 2 ) is shown separately. This figure consitutes the main result of the present Letter, and allows the contributions of the different vibrational $\mathrm{C} 1 \mathrm{~s}^{-1}$ intermediate states to the Auger spectrum to be traced separately. In photodouble-ionization spectra, the region of final state energies between 41.5 and $42.5 \mathrm{eV}$ is a blend of transitions into the $a$, the $b$ and the $X^{3} \Pi$ state [12,23]. Auger decay tends to populate the triplet states to a lesser extent. The series of the first five peaks at binding energies of $41.45(2) \mathrm{eV}$ and higher coincides quite well with those parts of the doubleionization spectrum assigned to the $a\left(1^{1} \Sigma^{+}\right)$state. The energy differences of our observed peaks within the experimental error agree with theoretical spectroscopic parameters of $\hbar \omega=238.1 \mathrm{meV}$ and $\hbar \omega x=2.1 \mathrm{meV}$ [12], if we assume that the observed maximum of the first peak is influenced by a contribution from the $X^{3} \Pi$ state. The shoulder below $41.5 \mathrm{eV}$ binding energy shows that the ground vibrational level of this state is populated, most probably from the $\nu^{\prime}=2$ intermediate state.

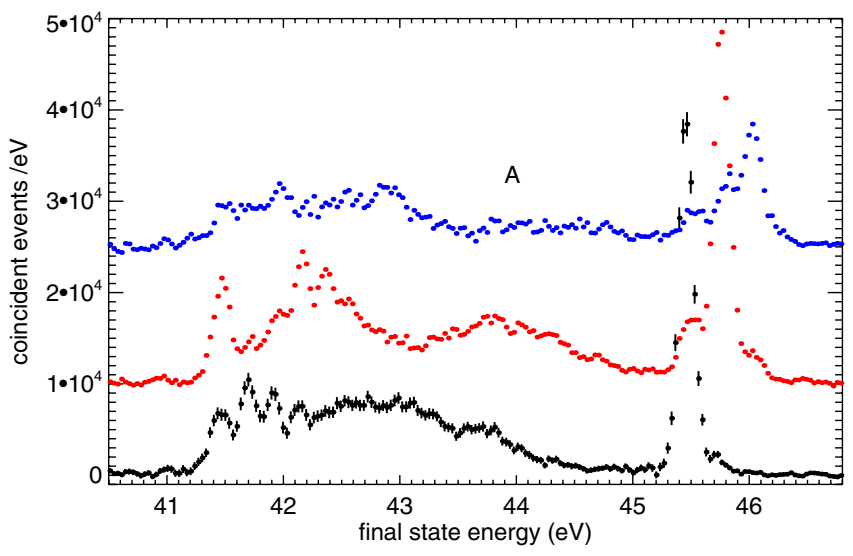

FIG. 2 (color online). Decay intensities from the $\mathrm{C} 1 s^{-1}, \nu^{\prime}=$ 0 (symbols), $\nu^{\prime}=1$ (red) and $\nu^{\prime}=2$ (blue) intermediate states. Intensities have been summed within a $150 \mathrm{meV}$ interval around the respective intensity maximum in the summed photoelectron curve (left-hand side panel of Fig. 1). Error bars shown for the $\nu^{\prime}=0$ curve are representative for all data. The curves for $\nu^{\prime}=$ 1 (red) and $\nu^{\prime}=2$ have been offset by 10000 and 25000 events/eV to improve visibility.
The next higher final state $b\left({ }^{1} \Pi\right)$, located at $R_{e}=$ $0.1250 \mathrm{~nm}$ [12], has a potential curve with a minimum far outside the FC region and is populated by transitions to final state energies from 41.757(10) eV [23] up to $44.5 \mathrm{eV}$. In this energy region higher vibrational levels of the $a$ state are also located, which make an unambiguous assignment complicated. How can this wide range of energies be explained? Since the potential curve descends steeply in the inner part of the FC region, it is there that high vibrational states can be populated. According to the spectroscopic parameters from [12], $\hbar \omega=184.5 \mathrm{meV}$ and $\hbar \omega x=2.1 \mathrm{meV}$, this would correspond to final states $\nu^{\prime \prime}=1-18$. The intensity pertaining to decay into these levels is not vibrationally resolved, which is probably due rather to the limited statistics of this data set than to the inherent limitations of our apparatus. Assigning the vibrationally resolved Auger spectra in Fig. 2 to these final states, we propose that the first two or three peaks belong to the $a$ state, but the unresolved hump at energies above $\approx 42.3 \mathrm{eV}$ belong mainly to $\nu^{\prime \prime}=3-13$ of the $b$ state. The deexcitation from $\nu^{\prime}=1$ shows a behavior similar to $\nu^{\prime}=$ 0 , but is shifted towards higher energies. As mentioned above the part below $42.6 \mathrm{eV}$ corresponds to $a$ and the intensity between 43.2 and $44.3 \mathrm{eV}$ may be correlated to $\nu^{\prime \prime}=9-15$ of the $b$ state.

The topmost data curve in Fig. 2, pertaining to intermediate states with $\nu^{\prime}=2$, show a more structured appearance in the binding energy region between 43.5 and $44.5 \mathrm{eV}$. From comparison with the photoionization spectra, we identify the three well resolved peaks at 43.73(2), 44.03(2) and 44.25(2) eV with the lowest three vibrational levels of the $A^{3} \Sigma^{+}$state. This state also is present in the $\nu^{\prime}=1$, but not in the $\nu^{\prime}=0$. As our method yields the Auger spectrum versus final state energy, not transition energy, states populated to a lesser extent can be identified more easily than in noncoincident spectra. This is the case for both the $X$ and the $A$ triplet states, which were neglected in earlier analyses of the Auger spectrum of $\mathrm{CO}[7,8]$.

Calculations $[12,13]$ show that the potential curve of the $d\left({ }^{1} \Sigma\right)$ state has a double well structure, with an inner minimum at $R_{e}=0.104 \mathrm{~nm}$, located well inside the Franck-Condon region, and an energy of $E_{\mathrm{fi}}=$ 45.461(3) eV. By a fit of Gaussian profiles to the three pronounced substates in the final state sum (top panel in Fig. 1), we arrive at vibrational spacings of 305(10) and 261(15) meV between $\nu^{\prime \prime}=0,1$ and $\nu^{\prime \prime}=1,2$, respectively. These values are near to the results from direct photo-double-ionization $(305 \pm 10$ and $278 \pm 10 \mathrm{meV})$ [12,24].

In a recent series of articles, Püttner et al. have developed a methodology for simultaneous lineshape fitting of noncoincident high resolution photoelectron spectra and the corresponding Auger electron spectra [4,5]. Their approach is based on a standard two-step formulation of the two electron transition amplitude, in which the parame- 
trized potential curves of the participating states and the electronic matrix elements are used as adjustable parameters. Lifetime vibrational interference [7] is included. As the number of adjustable parameters is large and the influence of each of them on the simulated spectra is diminished by the summation over all intermediate states, noncoincident spectra of very high quality and a meticulous data analysis are necessary for this approach to succeed. Moreover, the influence of satellite Auger decays on the noncoincident Auger spectra cannot be distinguished [15,25]. Results recently presented for CO [5] corroborate most theoretical findings about the singly and doubly ionized states involved $[2,12,13,17]$. A simulated photoelectronAuger electron coincidence map (Fig. 5a in [5]) shows considerable similarity with our experimental data. Closer inspection reveals, however, differences in the population of vibrationally excited levels of the $d\left({ }^{1} \Sigma\right)$ state. While Püttner $e t$ al. predict Auger decay practically only between levels of equal vibrational quantum number, in our experimental spectra we see significant transitions from $\nu^{\prime}=1$ to $\nu^{\prime \prime}=0$, and from $\nu^{\prime}=2$ to $\nu^{\prime \prime}=1,0$ (Fig. 2). The vibrational quantum energy of the $d\left({ }^{1} \Sigma\right)$ state is given as $319.7(15) \mathrm{meV}$ in [5], with a vanishing anharmonicity, in contrast to the lower values found in our experiment. As in the noncoincident Auger spectrum the main transitions into the $d\left({ }^{1} \Sigma\right)$ vibrational states all overlap at practically the same kinetic energy, their reliable separation is only possible when the Auger transitions can be plotted vs final state energy. This demonstrates the need for a direct experimental approach to these quantities.

Energy conservation even holds within a single photoelectron line between well defined vibrational states. In a two-dimensional map, as in Fig. 1, one consequence of this is the absence of lifetime broadening along the final state energy axis (top panel of the figure). While in the present experiment the apparatus broadening is still too large to allow subnatural linewidth spectroscopy [26], this is indeed possible with the method presented here, and will allow us to understand core level photoelectron and Auger spectra in far more detail than at present. We note that in an experiment with very small instrumental broadening, coincidence features presented in a two-dimensional map, as in Fig. 1, will take the shape of short dashes along the lines of constant final state energy [27]. By the apparatus broadening of the photoelectron and the Auger electron analyzer, these dashes can be blurred to an elliptic shape, which we will discuss in a publication about the instrumentation [21].

In conclusion, we have detected the electrons from normal Auger decay in $\mathrm{CO}$ after $\mathrm{C} 1 s$ photoionization. By our energy-resolved coincidence measurement each Auger electron could be correlated with its associated photoelectron. How the population of the Auger final states derives from the vibrationally resolved core-ionized intermediate states has been investigated in detail. Our method is a general one and has great promise for the investigation of sequential double ionization in molecules and clusters.
This work has been supported by the Deutsche Forschungsgemeinschaft and the Fonds der chemischen Industrie.

*Present address: IPP, c/o BESSY, Albert-Einstein-Str. 15, 12489 Berlin, Germany. uwe.hergenhahn@ipp.mpg.de

[1] K. Siegbahn, Rev. Mod. Phys. 54, 709 (1982).

[2] U. Hergenhahn, J. Phys. B 37, R89 (2004).

[3] L.S. Cederbaum, P. Campos, F. Tarantelli, and A. Sgamellotti, J. Chem. Phys. 95, 6634 (1991).

[4] R. Püttner et al., Phys. Rev. A 65, 042505 (2002).

[5] R. Püttner, X.-J. Liu, H. Fukuzawa, T. Tanaka, M. Hoshino, H. Tanaka, J. Harries, Y. Tamenori, V. Carravetta, and K. Ueda, Chem. Phys. Lett. 445, 6 (2007).

[6] K. J. Randall, A. L. D. Kilcoyne, H. M. Köppe, J. Feldhaus, A. M. Bradshaw, J.-E. Rubensson, W. Eberhardt, Z. Xu, P. D. Johnson, and Y. Ma, Phys. Rev. Lett. 71, 1156 (1993).

[7] N. Correia et al., J. Chem. Phys. 86, 5221 (1987).

[8] S. Sundin et al., Phys. Rev. A 58, 2037 (1998).

[9] J. Viefhaus, U. Hergenhahn, R. Hentges, A. Rüdel, and U. Becker in Physics of Electronic and Aomic Collisions: XXI International Conference, Abstr. of Contrib. Papers, Sendai, 1999, edited by Y. Itikawa et al., (unpublished), reprinted in U. Becker, J. Electron Spectrosc. Relat. Phenom. 112, 47 (2000); S. K. Semenov et al., Phys. Rev. A 75, 032707 (2007).

[10] R. Guillemin et al., Phys. Rev. Lett. 87, 203001 (2001).

[11] T. Weber et al., Phys. Rev. Lett. 90, 153003 (2003).

[12] J. H. D. Eland et al., J. Phys. B 37, 3197 (2004).

[13] T. Šedivcová, J. Fišer, P. R. Ždánská, and V. Špirko, J. Chem. Phys. 124, 214303 (2006).

[14] H. W. Haak, G. A. Sawatzky, and T. D. Thomas, Phys. Rev. Lett. 41, 1825 (1978).

[15] M. Neeb, J.-E. Rubensson, M. Biermann, and W. Eberhardt, J. Phys. B 29, 4381 (1996).

[16] CRC Handbook of Chemistry and Physics, edited by D. R. Lide (CRC Press, Boca Raton, 1996), 76th ed.

[17] T. X. Carroll et al., J. Chem. Phys. 116, 10221 (2002).

[18] F. Senf et al., Nucl. Instrum. Methods Phys. Res., Sect. A 467, 474 (2001).

[19] O. Kugeler, S. Marburger, and U. Hergenhahn, Rev. Sci. Instrum. 74, 3955 (2003).

[20] V. Myrseth et al., J. Electron Spectrosc. Relat. Phenom. 122, 57 (2002).

[21] O. Kugeler, V. Ulrich, A.M. Bradshaw, and U. Hergenhahn et al. (to be published).

[22] Y. Hikosaka et al., Phys. Rev. Lett. 98, 183002 (2007).

[23] M. Hochlaf et al., Chem. Phys. 207, 159 (1996).

[24] G. Dawber et al., J. Phys. B 27, 2191 (1994).

[25] A. Kivimäki, G. Snell, R. Hentges, M. Wiedenhöft, F. Heiser, O. Gessner, and U. Becker, Phys. Rev. A 54, 2137 (1996).

[26] J. Viefhaus, G. Snell, R. Hentges, M. Wiedenhöft, F. Heiser, O. Gessner, and U. Becker, Phys. Rev. Lett. 80, 1618 (1998)

[27] Under the assumption of long lived final states. 